\title{
Robust boson dispenser: Quantum state preparation in interacting many-particle systems
}

\author{
Irina Reshodko, ${ }^{*}$ Albert Benseny, and Thomas Busch \\ Okinawa Institute of Science and Technology Graduate University, 904-0495 Onna, Okinawa, Japan
}

(Received 8 March 2017; published 3 August 2017)

\begin{abstract}
We present a technique to control the spatial state of a small cloud of interacting particles at low temperatures with almost perfect fidelity using spatial adiabatic passage. To achieve this, the resonant trap energies of the system are engineered in such a way that a single, well-defined eigenstate connects the initial and desired states and is isolated from the rest of the spectrum. We apply this procedure to the task of separating a small pre-defined number of particles (up to 10) from an initial cloud and show that it can be implemented in radio-frequency traps using experimentally realistic parameters.

DOI: 10.1103/PhysRevA.96.023606
\end{abstract}

\section{INTRODUCTION}

Small samples of ultracold atoms trapped in external potentials are shaping up to become paradigmatic systems for exploring the fundamental building blocks of quantum many-body dynamics [1-5]. While in the weakly interacting regime samples with more than five atoms are well described by a mean-field approach $[2,6]$, strongly interacting systems have been shown to allow for the creation of highly correlated quantum many-body states [7,8]. Understanding and controlling interactions and many-particle dynamics is therefore crucial for accessing a larger part of these systems' Hilbert space.

One important ingredient in this quest is the development of high-fidelity quantum engineering techniques. This, however, is a nontrivial task, due to the large number of degrees of freedom present in many-particle systems, which make it hard to follow or reach specific states. It is therefore sensible to start the development with systems with only a small number of particles and later generalize the developed tools to larger systems.

One of the techniques which allow for high-fidelity state preparation in external potentials is spatial adiabatic passage (SAP) [9]. It is an analog to the well-known STIRAP technique in atomic physics [10-12] and utilizes the existence of a specific "dark" eigenstate to coherently transfer single particles between two localized spatial states [13]. Compared to STIRAP, the SAP setting can possess a larger variety of degrees of freedom, which has in recent years allowed one to extend the technique to multiple dimensions [14-16], angular momentum states [17,18], nonlinear systems [14,19-21], and interacting particles [22-26].

In this work we will extend the previous developments on interacting systems and show that the typical control that exists in ultracold atom experiments can be used to devise techniques based on SAP for the engineering of specific many-particle states. For this we will investigate the possibility of creating a single-particle source from two- and three-particle samples. We will also discuss the generalization of the proposed method to engineer the separation of a single particle from an arbitrary initial number of interacting particles, as well as the separation of an arbitrary, but well-defined, number of particles from the

*irina.reshodko@ oist.jp initial system. We also discuss a realistic implementation of the suggested protocol using radio-frequency traps.

Our manuscript is structured as follows. In order to understand the proposed protocols, we first briefly review the main ideas of the single-particle and two-particle SAP protocols in Sec. II. Then, in Sec. III, we present the modified SAP protocols designed for the separation of a specified number of particles from an atomic cloud and discuss their limits. To show that our ideas are realistic, we present in Sec. IV a study of a potential implementation of the particle separation protocol using radio-frequency traps. Finally, we conclude in Sec. V.

\section{SINGLE-PARTICLE AND TWO-PARTICLE SAP}

The fundamental principle behind SAP can be illustrated by considering a one-dimensional model in which a single particle is trapped in an external potential consisting of three truncated harmonic traps (see Fig. 1) [13]:

$$
V(x)=\frac{1}{2} m \omega^{2} \min \left[\left(x+d_{12}(t)\right)^{2}, x^{2},\left(x-d_{23}(t)\right)^{2}\right] .
$$

Here $d_{12}$ and $d_{23}$ are the distances between the minima of the left and middle traps and the middle and right traps, respectively, $m$ is the mass of the particle, and $\omega$ is the trapping frequency, which is taken to be identical for all three traps. Assuming that the particle is initially in the center-of-mass ground state of the left trap and that the evolution is carried out adiabatically, this system can be modeled by considering only the ground states of the three traps, $|1\rangle,|2\rangle$, and $|3\rangle$. The Hamiltonian of such a three-level system can then be written as

$$
\hat{H}(t)=\hbar\left(\begin{array}{ccc}
0 & \Omega_{12}(t) & 0 \\
\Omega_{12}(t) & 0 & \Omega_{23}(t) \\
0 & \Omega_{23}(t) & 0
\end{array}\right),
$$

where the $\Omega_{i j}(t)$ are the coupling frequencies between the states $|i\rangle$ and $|j\rangle$ which depend on the distance between the traps $d_{i j}$ for $i, j=1,2,3$. Diagonalizing this Hamiltonian gives one eigenstate, the so-called dark state, with zero eigenvalue, which only has contributions from the traps on the left and on the right,

$$
|D(\theta)\rangle=\cos \theta|1\rangle-\sin \theta|3\rangle, \quad \tan \theta=\frac{\Omega_{12}}{\Omega_{23}} .
$$




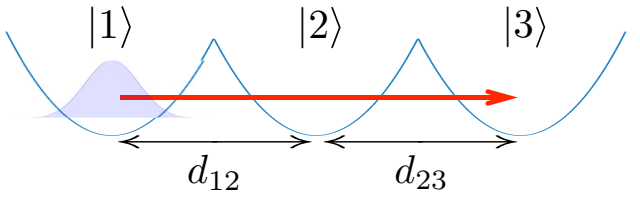

FIG. 1. Schematic of the SAP setup using a triple harmonic trap system. The ground states of the left, middle, and the right trap are given by $|1\rangle=|100\rangle,|2\rangle=|010\rangle$, and $|3\rangle=|001\rangle$, respectively. The distances $d_{12}$ and $d_{23}$ between the traps can be changed independently and the goal is to achieve a high-fidelity transfer of a particle from the left trap to the right one.

SAP then consists of following the dark state and transferring a particle from the left trap to the right by adiabatically changing $\theta$ from 0 to $\pi / 2$. This requires one to change the relative coupling strengths between the traps, which can be done by approaching and separating individual pairs. The movement sequence is famously counter-intuitive because the right and the middle traps, which are both empty, approach each other before the left trap starts moving. Since the process does not depend on the exact form of the positioning sequence, it is robust to experimental uncertainties.

The introduction of interactions to the system yields a loss of resonance in the tunneling process which, in principle, requires careful and time-dependent trapping potential adjustments $[14,19,21]$. However, it was recently shown that these adjustments are not necessary in few-particle systems for a large range of interaction strengths [24]. The Hamiltonian for $N$ ultracold bosons of mass $m$ in one dimension can be written as

$$
\hat{H}=\sum_{j=1}^{N}\left[-\frac{\hbar^{2}}{2 m} \frac{\partial^{2}}{\partial x_{j}^{2}}+V\left(x_{j}\right)\right]+g \sum_{k>j} \delta\left(x_{j}-x_{k}\right),
$$

where $V(x)$ is again given by Eq. (1) and the interaction between the particles is assumed to be pointlike with strength $g[27,28]$. To understand the underlying principles of how SAP works in interacting systems, which also are the key to understanding the techniques in the following section, we will briefly review the two-particle case here. It is described by the explicit Hamiltonian,

$$
\hat{H}=-\frac{\hbar^{2}}{2 m} \frac{\partial^{2}}{\partial x_{1}^{2}}-\frac{\hbar^{2}}{2 m} \frac{\partial^{2}}{\partial x_{2}^{2}}+V\left(x_{1}\right)+V\left(x_{2}\right)+g \delta\left(x_{1}-x_{2}\right),
$$

and the strength $g$ directly relates to the spectrum for two particles in a harmonic trap as [29]

$$
g=-\frac{2 \sqrt{2} \Gamma\left(1-E_{g} /(2 \hbar \omega)\right)}{\Gamma\left(\left(1-E_{g} /(\hbar \omega)\right) / 2\right)},
$$

where $\Gamma(E)$ is the gamma function. Thus, we can define an interaction energy $U_{\text {int }}$ as

$$
U_{\text {int }}=E_{g}-2 E_{0},
$$

where $E_{0}=\hbar \omega / 2$ is the (single-particle) harmonic oscillator ground-state energy and $E_{g}$ is the two-particle ground-state energy. In this and the following section we will use natural units where $\hbar=m=\omega=1$.
The existence of a range of intermediate interaction strengths where high-fidelity SAP transfer can take place can then be understood from the band structure of the Hamiltonian spectrum [24]. The lowest energy band has energies around 1 and contains states where the two atoms are in the ground states of different traps. The second band, which lies around energy values between 1 and 2 (depending on the interaction strength), corresponds to states where both particles are in the same trap, and contains a dark state similar to the one in Eq. (3) which allows for the transport of the particle pair. Higher bands correspond to states where at least one of the atoms is in an excited trap state. For those intermediate interaction strengths where the second band remains isolated from the other two bands, the dark state can be adiabatically followed and high-fidelity SAP transport can be achieved [24].

In this regime of intermediate interactions, the two particles are repulsively bound and effectively behave like a single particle [30]. It is therefore clear that all single-particle protocols transfer directly to this situation, and in particular it is straightforward to engineer a two-particle NOON state by changing $\theta$ from 0 to $\frac{\pi}{4}$. This leaves the system in the state $\frac{1}{\sqrt{2}}(|200\rangle-|002\rangle)$, with $|200\rangle$ and $|002\rangle$ denoting states with two particles in the left and in the right trap, respectively. NOON states are maximally entangled and are important in quantum engineering and quantum metrology [31,32], as they allow for phase measurements that can reach the fundamental Heisenberg limit [33].

\section{PARTICLE SEPARATION}

In the following we will discuss a process based on SAP which is not a straightforward generalization of a singleparticle protocol, but which allows one to split an initial many-particle state in a controlled manner.

\section{A. Two-particle case}

To demonstrate the principle of the process, let us initially assume that the left trap contains two particles only, and that the target state of the process has one particle in the left and the other in the right trap,

$$
\left|\psi_{\mathrm{i}}\right\rangle=|200\rangle \rightarrow\left|\psi_{\mathrm{f}}\right\rangle=\mid \begin{array}{lll}
1 & 01 & 1
\end{array} .
$$

Due to the atomic interactions, the initial and the final state have different energies and are in different energy bands. To make the desired coupling possible, it will therefore be necessary to match the energies and compensate for the absence of the interaction energy in the final state by adjusting the energies of the traps. This can be done in a time-independent manner by raising the energies of the middle and the right traps by $V_{\text {lift }}=U_{\text {int }}$ (or lowering in the case of attractive interactions), which ensures resonance between states $|200\rangle,|101\rangle$, and $|110\rangle$. This also energetically separates them from all other states, making the system effectively a three-level system, analogous to Eq. (2) (see Fig. 2). A darklike energy eigenstate, which involves only the initial and target states can then be found and the counterintuitive positioning sequence leads to the desired particle separation. 


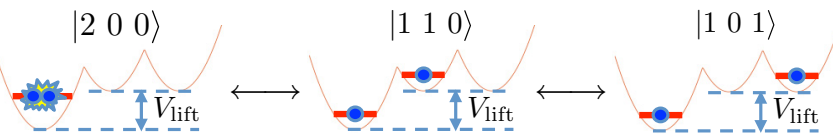

FIG. 2. Schematic of the three-level model for particle separation. Simultaneous lift of the right and the middle harmonic traps by $V_{\text {lift }}=$ $U_{\text {int }}$ makes the three states depicted resonant.

To confirm that the splitting process works as expected we will in the following simulate the above system first with truncated harmonic traps as external potential. Using the full Hamiltonian given in Eq. (5), we calculate the fidelity $F=$ $\left|\left\langle\psi_{\mathrm{f}} \mid \psi_{\mathrm{T}}\right\rangle\right|^{2}$ of the process, which is shown in Fig. 3(b) for $U_{\text {int }} \in\left[-\frac{1}{2}, 1\right]$ (solid blue line). Although there is a prominent dip in the weakly interacting regime, the process can be seen to result in high fidelities over a wide range of interaction strengths. This is easy to understand in the limit of infinitely
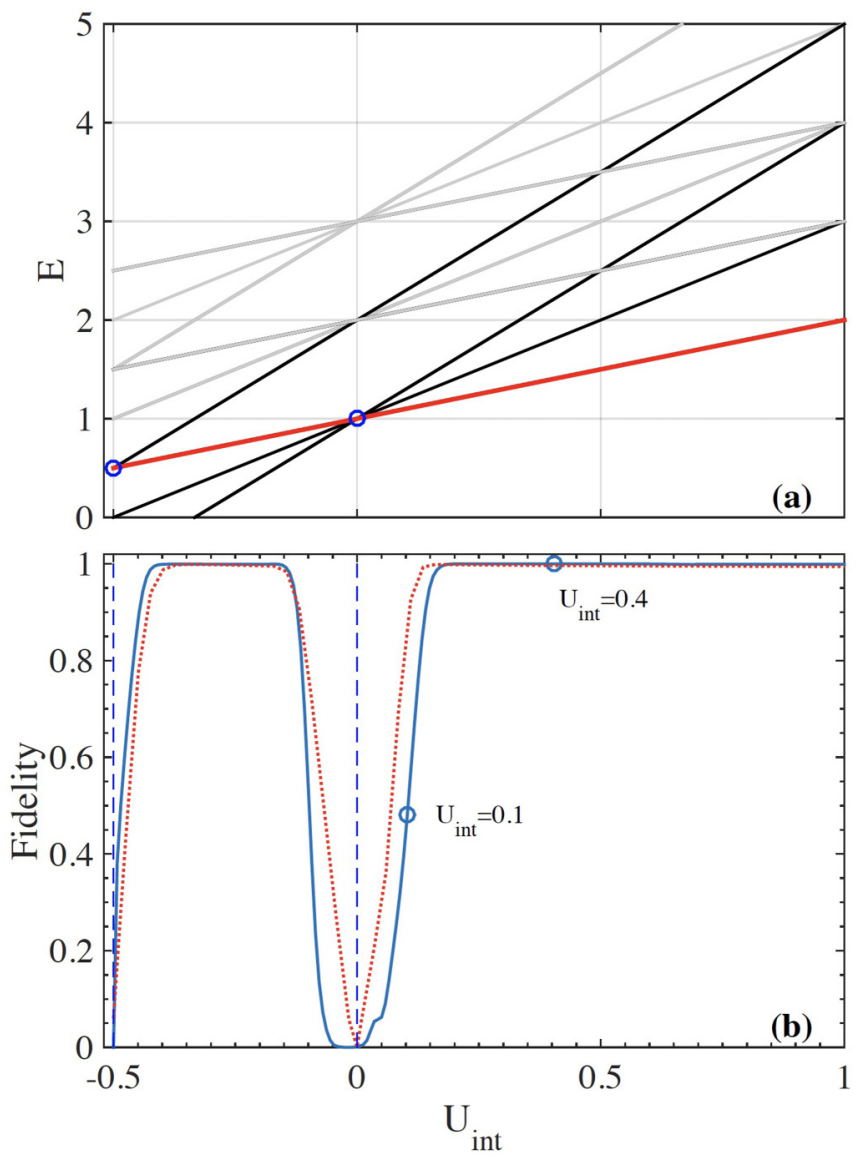

FIG. 3. (a) Bose-Hubbard spectrum of the two-particle Fock states in the three-trap system with only the lowest two energy levels in each trap considered and $V_{\text {lift }}=U_{\text {int }}$. The three degenerate states $|200\rangle,|110\rangle$, and $|101\rangle$ are in the band colored in red and additional degeneracies can be seen to appear at $U_{\text {int }}=0$ and $U_{\text {int }}=-1 / 2$. (b) Fidelities of the particle separation process as a function of the interaction energy, obtained using the full Hamiltonian time evolution (solid blue line) and BH model (dashed red line). Degeneracies of the spectrum appear at points marked as vertical dashed blue lines. The circles indicate interaction energy values which are analyzed in more details in Fig. 4. The energies $E$ and $U_{\text {int }}$ are given in units of $\hbar \omega$.

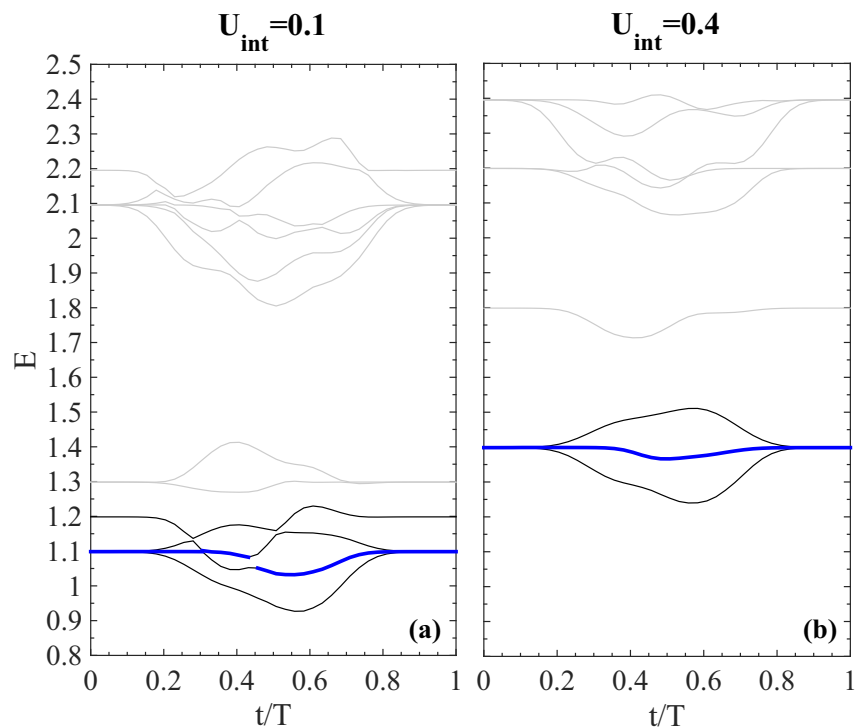

FIG. 4. Evolution of the energy spectrum during the particle separation process for (a) $U_{\text {int }}=0.1$ and (b) $U_{\text {int }}=0.4$. The darklike state is highlighted in blue. $E$ and $U_{\text {int }}$ are given in units of $\hbar \omega$.

repulsive interactions (the Tonks-Girardeau limit, $U_{\text {int }}=1$ ), where the bosonic particles can be described as noninteracting fermions [34]. The system can then be thought of as forming a Fermi sea in the harmonic trap, and by choosing $V_{\text {lift }}=1$ only the particle at the Fermi edge can tunnel. It is therefore natural to expect high-fidelity particle separation in this case.

The drop of fidelity in the weakly interacting regime can be understood by looking at the time-dependent spectrum of the Hamiltonian (5), which we show in Fig. 4 for two different values of $U_{\text {int }}$ [corresponding to the points indicated in Fig. 3(b)]. One can see that for weak interactions $\left(U_{\text {int }}=0.1\right)$ the lowest band, which contains the dark state, overlaps with the next higher lying one and therefore level crossings lead to the degraded fidelity. For stronger interactions $\left(U_{\text {int }}=0.4\right)$, the band overlap vanishes and following the dark state becomes possible. The drop of fidelity for $U_{\text {int }}=-\frac{1}{2}$ will be discussed below, in Sec. III B.

In the following sections we will extend the separation idea discussed above to systems with larger numbers of particles, $N$. However, since the resources required to diagonalize and numerically integrate the Schrödinger equation using the full Hamiltonian (4) scale exponentially with $N$, we will in the next section introduce a Bose-Hubbard (BH) model for this three-trap system. To establish correspondence, we will first compare the above results for the two-particle case to the two-particle BH model and then use the BH model to simulate the three-particle case.

\section{B. Bose-Hubbard model}

Let us assume a system of $N$ bosons distributed over three traps, which are lifted by energy values $V_{1}, V_{2}$, and $V_{3}$ (counted from left to right) with $m_{\mathrm{L}}$ vibrational states in each trap. Such a system contains $\left(\begin{array}{c}N+3 m_{\mathrm{L}}-1 \\ 3 m_{\mathrm{L}}-1\end{array}\right)$ Fock states and we associate each state with a matrix $\left\{n_{j i}\right\}$, where $n_{j i}$ is the number of particles 
in the $j$ th energy level of the $i$ th trap,

$$
\left|\left\{n_{j i}\right\}\right\rangle=\left|\begin{array}{ccc}
n_{01} & n_{02} & n_{03} \\
& \cdots & \\
\cdots & n_{j i} & \ldots \\
& \cdots & \\
n_{\left(m_{\mathrm{L}}-1\right) 1} & n_{\left(m_{\mathrm{L}}-1\right) 2} & n_{\left(m_{\mathrm{L}}-1\right) 3}
\end{array}\right| .
$$

Summing over all states and occupation numbers gives the overall number of particles, $\sum_{i=1}^{3} \sum_{j=0}^{m_{\mathrm{L}}-1} n_{j i}=N$. For states in which only the lowest band is occupied we use the more intuitive notation,

$$
\left|\left\{n_{0 i}\right\}\right\rangle=\left|n_{01} n_{02} n_{03}\right\rangle .
$$

The BH Hamiltonian for this system can then be written as

$$
\begin{aligned}
\hat{H}_{\mathrm{BH}}= & \hbar \omega \sum_{j=0}^{m_{\mathrm{L}}-1}\left(j+\frac{1}{2}\right) \hat{N}_{j}^{\text {level }}+\sum_{i=1}^{3} V_{i} \hat{N}_{i}^{\text {trap }} \\
& +\frac{U_{\text {int }}}{2} \sum_{i=1}^{3} \hat{N}_{i}^{\text {trap }}\left(\hat{N}_{i}^{\text {trap }}-1\right)+H_{\text {tunnel }},
\end{aligned}
$$

where the $a_{i j}$ are the annihilation operators for a boson in the $j$ th level of trap $i$ and the $\hat{n}_{j i}=\hat{a}_{j i}^{\dagger} \hat{a}_{j i}$ are their associated particle number operators. The total number of particles in the $i$ th trap is therefore

$$
\hat{N}_{i}^{\text {trap }}=\sum_{j=0}^{m_{\mathrm{L}}-1} \hat{n}_{j i},
$$

with corresponding eigenvalues $N_{i}^{\text {trap }}$ and the total number of particles in the $j$ th band is

$$
\hat{N}_{j}^{\text {level }}=\sum_{i=1}^{3} \hat{n}_{j i} .
$$

with eigenvalues $N_{j}^{\text {level }}$. The first two terms of Eq. (11) correspond to the single-particle eigenenergies of the atoms in their respective levels, the third term describes the particleparticle interactions, and the last term accounts for all events where $p$ particles tunnel between two adjacent traps (the details on how to calculate the respective tunnel couplings are given in the appendix).

When the traps are far apart and the tunneling couplings are negligible it is sufficient to consider only the first three terms of the Hamiltonian in Eq. (11), which has the Fock states $\left|\left\{n_{j i}\right\}\right\rangle$ as its eigenstates. Their associated energies, $E_{\text {total }}\left(\left\{n_{j i}\right\}\right)$, then depend only on the interaction $U_{\text {int }}$ and potential lifts $V_{i}$. In particular, for states in the lowest band, $\left|n_{01} n_{02} n_{03}\right\rangle, E_{\text {total }}$ reduces to

$$
E_{\text {total }}\left(\left\{n_{0 i}\right\}\right)=\frac{N}{2} \hbar \omega+\sum_{i=1}^{3} V_{i} n_{0 i}+\frac{U_{\text {int }}}{2} \sum_{i=1}^{3} n_{0 i}\left(n_{0 i}-1\right) .
$$

The resonance condition between two Fock states, $\left|\left\{n_{j i}\right\}\right\rangle$ and $\left|\left\{n_{j i}^{\prime}\right\}\right\rangle$, can be written as

$$
E_{\text {total }}\left(\left\{n_{j i}\right\}\right)=E_{\text {total }}\left(\left\{n_{j i}^{\prime}\right\}\right),
$$

which can be used to find the appropriate trap lifts to create the SAP triplet of resonant states $\left(|200\rangle,|110\rangle\right.$, and $\left.\mid \begin{array}{lll}1 & 0 & 1\rangle\end{array}\right)$ for the particle separation protocol used above:

$$
V_{1}=0 ; \quad V_{\text {lift }}=V_{2}=V_{3}=U_{\text {int }} .
$$

The fidelities for the SAP separation process obtained from this BH model can be seen in Fig. 3(b) to be very similar to the ones obtained using the full Hamiltonian. Both approaches show large plateaus of high fidelity with drops around $U_{\text {int }}=0$ and $-\frac{1}{2}$. This can be understood in the $\mathrm{BH}$ model by examining the spectrum of the two-particle Fock states, considering only the lowest two Bloch bands $\left(m_{\mathrm{L}}=2\right)$, shown in Fig. 3(a). The highlighted red line corresponds to the energy of the degenerate SAP triplet and crossings between this band and other Fock states appear exactly at $U_{\text {int }}=0$ and $U_{\text {int }}=-1 / 2$. The drops in fidelity around these values can therefore be attributed to these level crossings and, in particular, for $U_{\text {int }}=-1 / 2$, the band that is crossed corresponds to the one containing states where one particle is in the ground state of the left trap and the other is in the first excited state of the middle or the right trap.

This demonstrates that the $\mathrm{BH}$ model reproduces the main features of the full model and we will be using it in the following to design and simulate particle separation processes in systems with larger particle numbers.

\section{C. $N$-particle case}

Starting with a cloud of $N$ particles initially located in the left trap, we will show in this section that it is possible to separate an arbitrary number of particles out of it. While the preparation of such an initial state is by today still experimentally challenging, recent progress in this direction has shown that this can be done for a wide range of particle numbers [1]. We will consider first the case where after the SAP dynamics exactly $M$ particles remain in the left trap, and later consider the case where exactly $M$ particles are separated into the right trap. The differences between these two cases will be explained below.

For the first case the initial and the target state are given by

$$
\left|\psi_{\mathrm{i}}\right\rangle=|N 00\rangle \rightarrow\left|\psi_{\mathrm{t}}\right\rangle=|M 0(N-M)\rangle,
$$

and the degeneracy conditions in Eqs. (14) and (15), lead to a simple formula for the trap lift,

$$
V_{1}=0 ; \quad V_{\text {lift }}=V_{2}=V_{3}=M U_{\text {int }} .
$$

It is important to note that this formula implies that it is not necessary to know the initial number of particles $N$ to keep the well-defined number $M$ of particles in the left trap, as $V_{\text {lift }}$ only depends on $M$.

Let us consider the case of $N=3$ with the target state

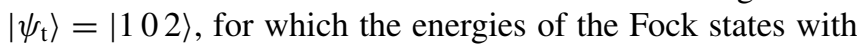
only the first two energy levels considered are shown in Fig. 5(a). One can immediately see that degeneracies appear at $U_{\text {int }}=\left\{-\frac{1}{3}, 0,1\right\}$, which correspond to drops in fidelity in the vicinity of these interaction energy values; see Fig. 5(c). As the spectrum will consist of more and more bands for increasing particle numbers, it is easy to see that the interaction region in which high-fidelity particle separation of the kind $|N 00\rangle \rightarrow|10(N-1)\rangle$ is possible, will become more and more fragmented due to additional crossings. 

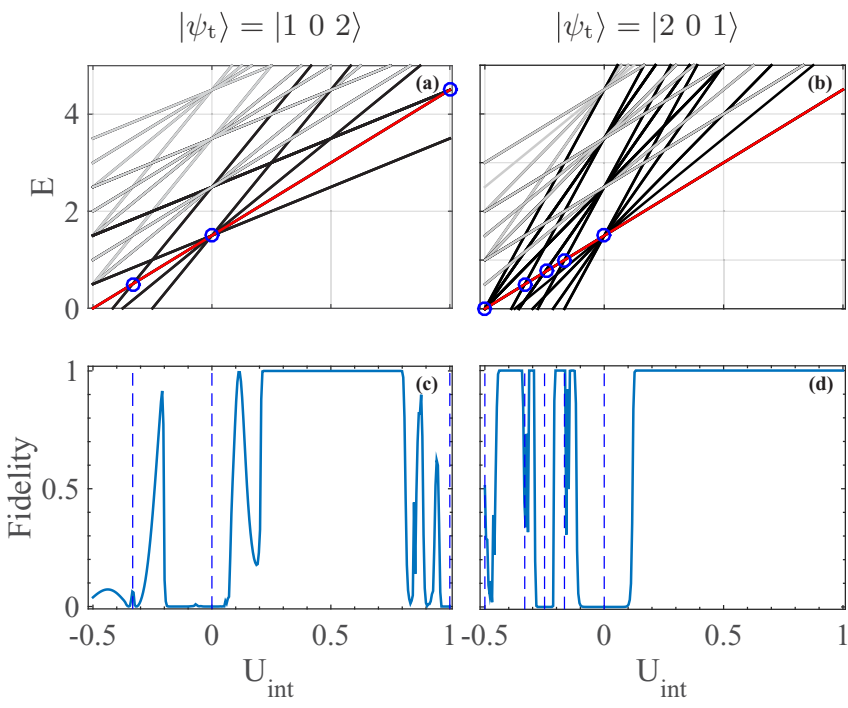

FIG. 5. (a) Energy spectrum of three-particle Fock states in the $\mathrm{BH}$ model for $m_{\mathrm{L}}=2$ for the target state $|102\rangle$ (and $V_{\text {lift }}=U_{\text {int }}$ ). The energy of the SAP triplet is highlighted in red. (c) Corresponding particle separation fidelities. (b) and (d) are the same as (a) and (c) but for $|201\rangle$ (and $V_{\text {lift }}=2 U_{\text {int }}$ ). The circles in the top row and vertical dashed lines in the bottom row indicate the positions where level crossings between the SAP triplet and other bands exist. The energies $E$ and $U_{\text {int }}$ are given in units of $\hbar \omega$.

If we consider the separation process which transfers a finite number of particles out of the original trap,

$$
\left|\psi_{\mathrm{i}}\right\rangle=|N 00\rangle \rightarrow\left|\psi_{\mathrm{t}}\right\rangle=|(N-M) 0 M\rangle,
$$

the lift required by Eqs. (14) and (15) is now given by

$$
V_{1}=0 ; \quad V_{\text {lift }}=V_{2}=V_{3}=(N-M) U_{\text {int }},
$$

and depends only on the number of separated particles $N-M$. While this is a complication, for $M=1$ the lift guarantees that the SAP triplet is always energetically isolated from other Fock states in the repulsive regime.

To see this we show in Fig. 5(b) the spectrum of the three-particle Fock states for $N=3$ and $M=1$ with $m_{\mathrm{L}}=2$. While there are multiple level crossings visible in the attractive interaction regime, the energy of the SAP triplet is the lowest for any repulsive value of $U_{\text {int }}$. This leads to a broad plateau in which the separation process gives high fidelities. Increasing the number of particles further leads to a denser and denser spectrum, but the SAP band remains the lowest, and therefore isolated, over the full repulsive interacting range, allowing the separation process for a single particle in principle to work for all possible initial particle numbers. It is worth noting though that the changes in the tunneling strengths between the traps during the dynamics of the SAP process results in nonzero bandwidths and therefore potential overlaps in denser spectra. This can lead to transitions out of the SAP triplet that lower the process fidelity. While in principle the bands can be kept arbitrarily narrow by decreasing the minimum approach distance between the traps, this would come at the price of having to increase the total time of the process, which is highly undesirable. It is therefore important to study the process in experimentally realistic settings.

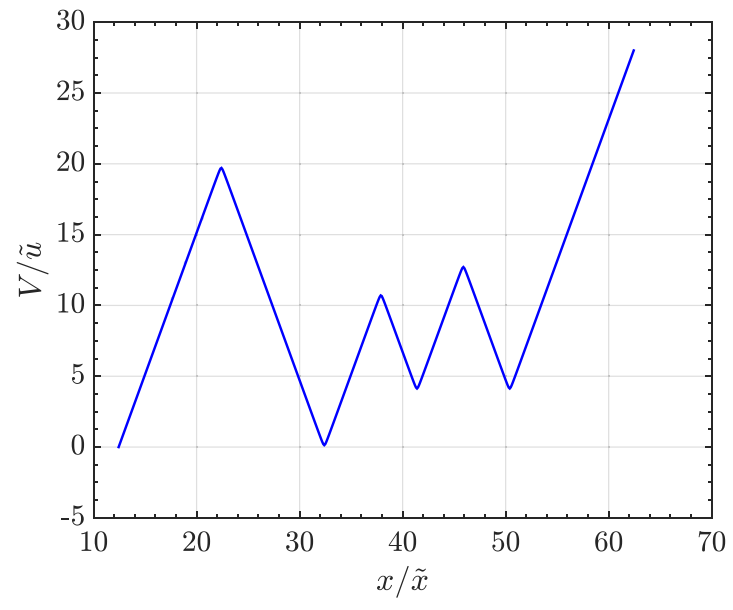

FIG. 6. Triple-well RF potential generated using six different frequencies and the parameters given in the text. The position of the left trap is fixed at $x_{1}=20 \tilde{x}$ and the corresponding RF is $\omega_{2}^{0}=\mu g_{F} b x_{1} / \hbar \approx 596 \mathrm{kHz}$. The maximum distance between the middle and the left or the right trap is $d=9 \tilde{x}$ and the corresponding minimum distance is $d_{\min }=6 \tilde{x}$. The difference between frequencies at time $t=0$ is $\Delta \omega=\mu g_{F} b d /(2 \hbar) \approx 134 \mathrm{kHz}$ and the frequency for the left trap edge is $\omega_{1}^{0}=\omega_{2}^{0}-2 \Delta \omega \approx 328 \mathrm{kHz}$. All the other frequencies are $\omega_{i}^{0}=\omega_{2}^{0}+(i-2) \Delta \omega, i=3, \ldots, 6$.

\section{RADIO FREQUENCY TRAPS}

While truncated harmonic potentials are very convenient for theoretical studies as they guarantee fulfillment of the resonance condition, they are experimentally unrealistic. We will therefore in the following examine a setup using radiofrequency (RF) traps to show that the process discussed above is viable as a quantum engineering technique. The physics of RF traps is well studied [35-37] and they are flexible tools that are available in many laboratories worldwide, making them ideal candidates to study the particle separation protocol.

\section{A. System}

We consider an atom with two hyperfine sublevels $\left(m_{F}=\right.$ $\left.\pm \frac{1}{2}\right)$ in an inhomogeneous magnetic field, $B(x)=b x$, which is irradiated by a linearly polarized RF field, $\vec{B}_{\mathrm{rf}} \cos (\omega t)$. In this setup the atom will experience an external potential with a minimum at position $x_{0}$ corresponding to the resonance condition $\mu g_{F} m_{F} b x_{0}=\hbar \omega$ [38], where $\mu \approx 9.27 \times 10^{-24} \mathrm{~A} / \mathrm{m}^{2}$ is the Bohr magneton and $g_{F}$ is the atomic $g$ factor. Using more than one RF allows one to create a multitrap potential [38] and a triple-well setup can be realized using six different frequencies [36] (see Fig. 6).

The external potential felt by the atom is then described by $[36,38]$

$$
V_{+}(x)=(-1)^{n(x)}\left[E_{+}(x)-\frac{\hbar \omega_{n(x)}}{2}\right]-\sum_{k=1}^{n(x)-1}(-1)^{k} \hbar \omega_{k},
$$

where

$$
\begin{aligned}
& E_{+}(x)=\frac{1}{2} \sqrt{\hbar^{2} \Omega^{2}+\left(\mu g_{F} b x-\hbar \omega_{n(x)}+2 L_{n(x)}(x)\right)^{2}}, \\
& L_{n}(x)=\sum_{j \neq n} \frac{\hbar^{2} \Omega^{2}}{4\left[\mu g_{F} b x-\hbar \omega_{j}\right]},
\end{aligned}
$$


and an integer $n(x)$ is chosen such that $\mu g_{F} b x-\hbar \omega_{n(x)}$ is minimized for all $x$, i.e., $n(x)$ is the label of the most relevant frequency at each point. The distance between the traps and their respective ground-state energies (which depend linearly on the trap lift) can be controlled by changing the $\omega_{i}$, which can be done with great precision.

For our simulations we use the following experimentally realistic parameters: the magnetic field gradient is chosen to be $b=-213 \mathrm{G} / \mathrm{cm}$, the atomic $g$ factor is $g_{F}=-\frac{1}{2}$, the Rabi frequency is $\Omega=2 \pi \times 0.5 \mathrm{kHz}$, and the mass of an ${ }^{87} \mathrm{Rb}$ atom is $m_{\mathrm{Rb}}=1.44 \times 10^{-25} \mathrm{~kg}$. For convenience we also scale all lengths by $\tilde{x}=\left(4 \hbar^{2} / \mu g_{F} b m_{\mathrm{Rb}}\right)^{\frac{1}{3}} \approx 3.18 \times 10^{-7} \mathrm{~m}$, time by $\tilde{t}=\left(16 \hbar m_{\mathrm{Rb}} /\left(\mu g_{F} b\right)^{2}\right)^{\frac{1}{3}} \approx 1.34 \times 10^{-4} \mathrm{~s}$, and energy by $\tilde{u}=m_{\mathrm{Rb}} \tilde{x}^{2} / \tilde{t}^{2} \approx 7.85 \times 10^{-31} \mathrm{~J}$. In these units, the TonksGirardeau regime is achieved at $U_{\mathrm{int}}^{\mathrm{TG}} \approx 1.64 \tilde{u}$.

\section{B. Particle separation}

In order to implement the SAP particle separation protocol, we use the following time dependencies of the RFs,

$$
\begin{aligned}
& \omega_{1}(t)=\omega_{1}^{0}-\frac{V_{\text {lift }}}{\hbar}, \\
& \omega_{2}(t)=\omega_{2}^{0}, \\
& \omega_{3}(t)=\omega_{3}^{0}+\frac{1}{2} f_{1}(t)+\frac{V_{\text {lift }}}{\hbar}, \\
& \omega_{4}(t)=\omega_{4}^{0}+f_{1}(t), \\
& \omega_{5}(t)=\omega_{5}^{0}+\frac{1}{2}\left(f_{1}(t)+f_{2}(t)\right), \\
& \omega_{6}(t)=\omega_{6}^{0}+f_{2}(t),
\end{aligned}
$$

where

$$
\begin{aligned}
f_{1}(t) & =-\frac{\mu g_{F} b d_{\text {min }}}{\hbar} f(t, 0), \\
f_{2}(t) & =-\frac{\mu g_{F} b d_{\min }}{\hbar}[f(t, 0)+f(t, \delta t)], \\
f(t, \delta) & = \begin{cases}\sin ^{2}\left(\frac{2 \pi(t-\delta)}{T}\right) & 0 \leqslant t-\delta<\frac{T}{2}, \\
0 & \text { otherwise. }\end{cases}
\end{aligned}
$$

The parameters $\delta t$ is the trap movement delay, $d_{\min }$ is the minimum distance between the middle and the left or the right traps, and $T$ is the total duration of the process.

For simplicity, and at variance with the truncated harmonic trap case in the previous section, where we raised the energy of the middle and right traps by $V_{\text {lift }}$, here we achieve the same effect by lowering the energy of the left trap by the same amount. This way, only one of the traps is affected by the energy shift.

We simulate the SAP separation process by starting with two atoms cooled to the ground state of the left trap with total energy $E_{g}$. Similarly to the truncated triple harmonic potential case, we calculate the energy lift value $V_{\text {lift }}=U_{\text {int }}=E_{g}-E_{0}$, where $E_{0}$ is the ground-state energy of two noninteracting atoms in the left trap. Both $E_{g}$ and $E_{0}$ are calculated numerically by direct diagonalization of the Hamiltonian. The

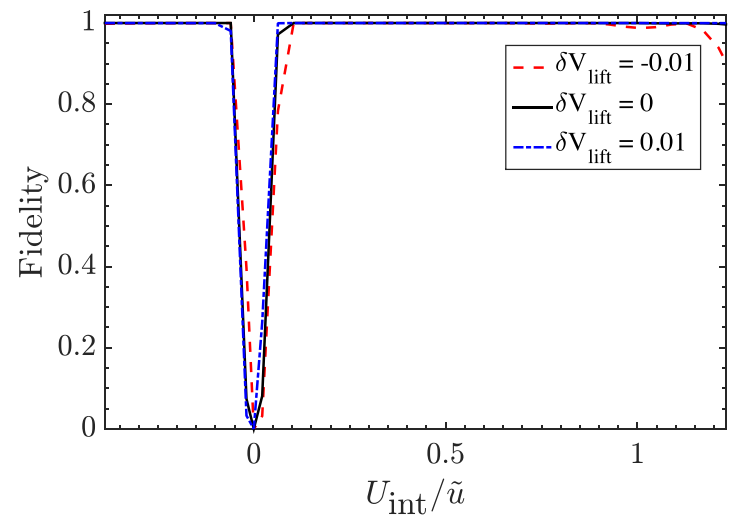

FIG. 7. Fidelity of the particle separation protocol in RF traps with perturbed maximum lift value. The fidelity results for very strong interactions up to the Tonks-Girardeau regime $U_{\mathrm{int}}^{\mathrm{TG}} \approx 1.64 \tilde{u}$ are not physical due to numerical error and are not shown on the plot.

solid line in Fig. 7 shows the resulting fidelities of the particle separation process and one can immediately see that for a wide range of repulsive interactions the process results in high-fidelity particle separation.

To account for possible experimental uncertainties in determining the interaction energy, we also show the particle separation fidelities for small errors in the energy shift in Fig. 7. We considered both negative and positive perturbation values $\delta V_{\text {lift }}$, and the effective energy shift value used in the simulation is calculated as $V_{\text {eff }}=V_{\text {lift }}+\delta V_{\text {lift }}$. One can see that the proposed implementation of the particle separation protocol is robust against small errors in the interaction energy measurements as well as against an imperfect execution of the lowering of the left trap. The robustness of the SAP protocol is discussed in more detail in [13].

It is important to note that the model we use to describe the RF traps is only valid when the RFs used are sufficiently far from each other [38]. When the frequencies come too close, the resulting potential becomes discontinuous and no longer describes the experimental situation. This limitation has been taken into account in our simulations by ensuring that the closest approach of two frequencies leads to a discontinuity smaller than $0.01 \tilde{u}$, which has a negligible effect on the dynamics. Furthermore, lowering the ground-state energy of the left trap requires one to adjust the change of the RFs $\omega_{1}$ and $\omega_{3}$. While this has an effect on the middle trap, it is also very small (on the order of $0.002 \tilde{u}$ ) and therefore also has no real effect on the process fidelity.

\section{Scaling with the number of particles}

Let us finally discuss the limits of the proposed protocol. Since for increasing numbers of particles the energy spectrum becomes more fragmented, it will be harder and harder to keep the system within the SAP triplet. To quantify the limit we determine the size of the maximum energy gap $\Delta E$ between the SAP triplet and the neighboring bands as a function of the initial and final number of particles in the left trap over the whole range of repulsive interactions. In addition we define the value of the interaction strength corresponding to this maximal energy gap $U_{\mathrm{int}}^{\mathrm{opt}}$, and the distance between the two points at 


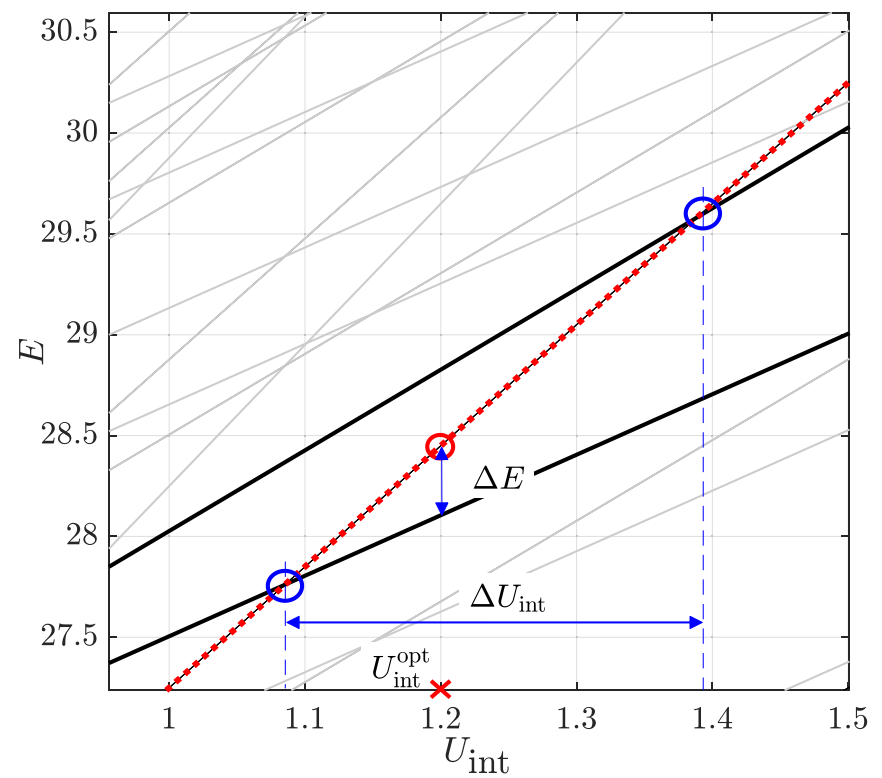

FIG. 8. Schematic indicating the definitions of $\Delta E, \Delta U_{\text {int }}$, and $U_{\text {int }}^{\text {opt }}$ using a Fock space energy spectrum. The dotted red line corresponds to the energy of the SAP triplet, and the black and gray lines show the energies of the other Fock states in the system (cf. Fig. 5). The blue circles indicate the points of intersection of the SAP triplet energy band with the closest, neighboring energy bands.

which the SAP triplet crosses other bands $\Delta U_{\text {int }}$ (see Fig. 8). Since at the point $U_{\mathrm{int}}^{\mathrm{opt}}$ the process works best, it gives a good insight for its limits: (1) $\Delta E$ quantifies how hard it is to follow the dark state and (2) $\Delta U_{\text {int }}$ indicates how fragmented the region, in which a high-fidelity process can be expected, has become.

The results reveal that the value of $\Delta E$ does not depend on the initial number of particles $N$, but only on the numbers of particles that are to be moved out of the trap, $N-M$. This can be easily understood by considering the structure of the energy spectrum (see Fig. 5). The energy band of each Fock state increases linearly with the interaction energy, intersecting $U_{\text {int }}=0$ at points that correspond to combinations of the excited trap eigenstates. The slope then depends on the number of particles in the right and the middle traps and it is easy to see that the $N-M=1$ energy band has the smallest slope, the $N-M=2$ has the second smallest slope, etc. With increasing $N-M$ one needs, of course, to include more energy levels in the model to account for all intersections, but the structure does not depend on $N$. However, $\Delta U_{\text {int }}$ rapidly decrease with increasing $N-M$ and in Fig. 9 we show our figures of merit. From there one can estimate that a realistic upper limit on $N-M$ is 10 particles, independent of the initial number of particles. Thus, for any $N$, by using, for example, Feshbach resonance [39-41], one can tune the interaction energy to access the region where the particle separation protocol is the easiest.

\section{CONCLUSIONS}

In this work we have proposed a protocol based on the spatial adiabatic passage technique that allows one to divide
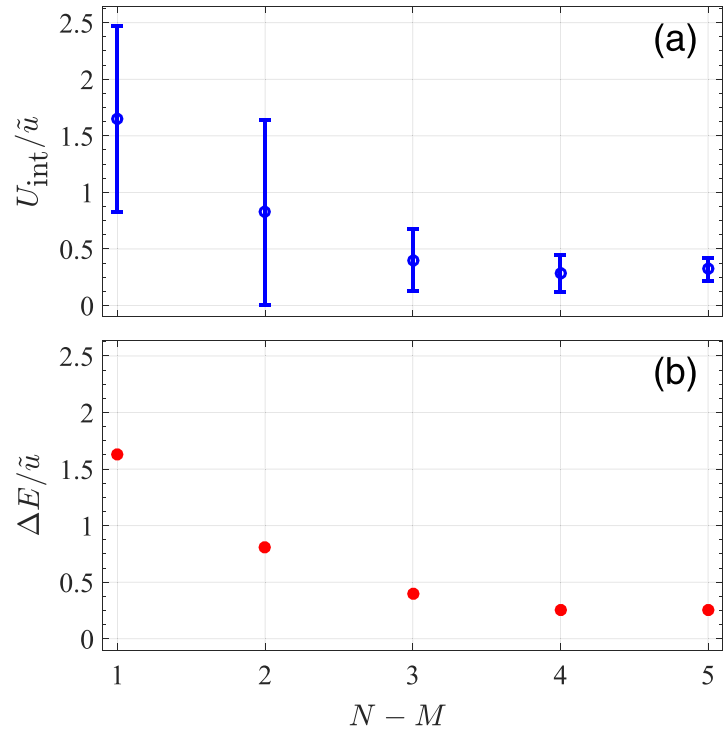

FIG. 9. (a) Optimal interaction energy value $U_{\text {int }}^{\text {opt }}$ with its margin $\Delta U_{\text {int }}$ as an error bar and (b) energy gap $\Delta E$. The Fock energy calculations are performed for RF traps using the Bose-Hubbard model for the three lowest energy bands.

a sample of interacting particles in a controlled way. The technique is based on engineering a quasi-three-level system by raising or lowering the energies of some of the traps and allowing for an adiabatic transition between initial and target states. We have explicitly examined the cases $|200\rangle \rightarrow$ $|101\rangle$ for a two-particle system and $|300\rangle \rightarrow|201\rangle$ for a three-particle system and shown that the SAP protocol results in high fidelities over large ranges of interaction energies. The regions where the protocol fails can be found from the level crossings present in the spectrum of a Bose-Hubbard model.

We have also shown that this protocol is realistic and robust against experimental uncertainties by examining a setting where two ${ }^{87} \mathrm{Rb}$ atoms were trapped in a radiofrequency trap setup. Using experimentally realistic parameters, the same high fidelities were obtained as for the idealized system, showing that quantum engineering techniques based on spatial adiabatic passage are useful for interacting particle systems. The protocol we proposed is independent of the number of initial particles and can be therefore used also in systems with large initial particle numbers.

\section{ACKNOWLEDGMENT}

This project was supported by the Okinawa Institute of Science and Technology Graduate University.

\section{APPENDIX: TUNNELING COUPLINGS}

The tunneling term in the BH Hamiltonian (11) is defined as 


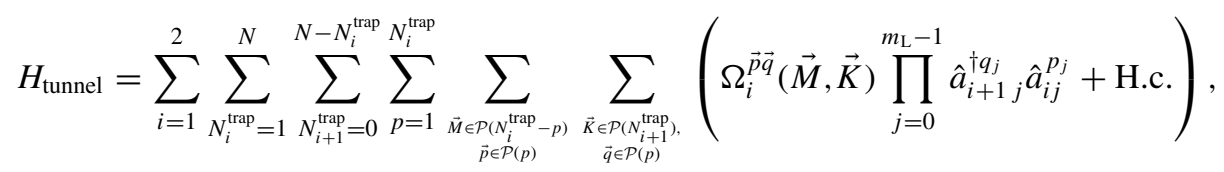

and it includes all tunneling events of $p$ particles between adjacent traps $i$ and $i+1$. The set $\mathcal{P}(n)$ contains all possible ways to distribute $n$ particles into $m_{\mathrm{L}}$ energy levels of one trap, and

$$
\sum_{j=0}^{m_{\mathrm{L}}-1} p_{j}=\sum_{j=0}^{m_{\mathrm{L}}-1} q_{j}=p
$$

The corresponding coupling coefficients $\Omega_{i}^{\vec{p} \vec{q}}(\vec{M}, \vec{K})$ denote the tunneling frequencies of $p$ atoms between the level occupation configurations $\vec{p}=\left(p_{0}, \ldots, p_{m_{\mathrm{L}}-1}\right)$ and $\vec{q}=$ $\left(q_{0}, \ldots, q_{m_{\mathrm{L}}-1}\right)$ of traps $i$ and $i+1$, respectively.

In what follows we derive the tunneling coupling amplitudes between two general Fock states,

$$
\left|\psi_{\mathrm{i}}\right\rangle_{i}^{\vec{p} \vec{q}}=\left|\begin{array}{cc}
M_{0 i}+p_{0} & K_{0(i+1)} \\
M_{1 i}+p_{1} & K_{1(i+1)} \\
\cdots & \cdots \\
M_{\left(m_{\mathrm{L}}-1\right) i}+p_{m_{\mathrm{L}}-1} & K_{\left(m_{\mathrm{L}}-1\right)(i+1)}
\end{array}\right|
$$

and

$$
\left|\psi_{\mathrm{t}}\right\rangle_{i}^{\vec{p} \vec{q}}=\left|\begin{array}{cc}
M_{0 i} & K_{0(i+1)}+q_{0} \\
M_{1 i} & K_{1(i+1)}+q_{1} \\
\cdots & \cdots \\
M_{\left(m_{\mathrm{L}}-1\right) i} & K_{\left(m_{\mathrm{L}}-1\right)(i+1)}+q_{m_{\mathrm{L}}-1}
\end{array}\right|
$$

which contain occupation numbers for traps $i$ and $i+1$. The coupling coefficient between these two states is defined from the general Hamiltonian (5) as

$$
\Omega_{i}^{\vec{p} \vec{q}}(\vec{M}, \vec{K})=\left\langle\psi_{\mathrm{t}}|\hat{H}| \psi_{\mathrm{i}}\right\rangle_{i}^{\vec{p} \vec{q}} .
$$

If $\Omega_{i}^{\vec{p} \vec{q}}(\vec{M}, \vec{K}) \neq 0$, then the corresponding relevant term that will appear in the $\mathrm{BH}$ Hamiltonian is proportional to $\prod_{j=0}^{m_{\mathrm{L}}-1} \hat{a}_{i+1}^{\dagger q_{j}} \hat{a}_{i j}^{p_{j}}$, thus

$$
\begin{aligned}
\Omega_{i}^{\vec{p} \vec{q}}(\vec{M}, \vec{K}) & =\tilde{\Omega}_{i}^{\vec{p} \vec{q}}\left\langle\psi_{\mathrm{t}}\left|\prod_{j=0}^{m_{\mathrm{L}}-1} \hat{a}_{i+1 j}^{\dagger q_{j}} \hat{a}_{i j}^{p_{j}}\right| \psi_{\mathrm{i}}\right\rangle_{i}^{\vec{p} \vec{q}} \\
& =\prod_{j=0}^{m_{\mathrm{L}}-1} \sqrt{\frac{\left(M_{j}+p_{j}\right) !}{M_{j} !} \frac{\left(K_{j}+q_{j}\right) !}{K_{j} !}} \tilde{\Omega}_{i}^{\vec{p} \vec{q}} .
\end{aligned}
$$

If $\vec{M}=\overrightarrow{0}$ and $\vec{K}=\overrightarrow{0}$, then

$$
\Omega_{i}^{\vec{p} \vec{q}}(\overrightarrow{0}, \overrightarrow{0})=\prod_{j=0}^{m_{\mathrm{L}}-1} \sqrt{p_{j} ! q_{j} ! \tilde{\Omega}_{i}^{\vec{p}} \vec{q}}
$$

where $\tilde{\Omega}_{i}^{\vec{p} \vec{q}}$ is the tunneling frequency of all $p$ atoms between level occupation configurations $\vec{p}$ of the trap $i$ and $\vec{q}$ of the empty trap $i+1$.

Since only the order of magnitude is important in order to show the shape of the regions of high-fidelity particle separation, we assume $\Omega_{i}^{\vec{p} \vec{q}}(\overrightarrow{0}, \overrightarrow{0}) \approx \tilde{\Omega}_{i}^{p}$. Here $\tilde{\Omega}_{i}^{p}$ is the tunneling frequency of $p$ atoms between the ground states of traps $i$ and $i+1$ in the absence of other atoms. In the three-particle calculations we assumed $\tilde{\Omega}_{i}^{3} \propto \tilde{\Omega}_{i}^{2}$, while $\tilde{\Omega}_{i}^{2}$ and $\tilde{\Omega}_{i}^{1}$ were calculated numerically. Equation (A5) can thus be written as

$$
\Omega_{i}^{\vec{p} \vec{q}}(\vec{M}, \vec{K}) \approx \prod_{j=0}^{m_{\mathrm{L}}-1} \sqrt{\frac{\left(M_{j}+p_{j}\right) !}{M_{j} !} \frac{\left(K_{j}+q_{j}\right) !}{K_{j} !} \frac{1}{p_{j} ! q_{j} !}} \tilde{\Omega}_{i}^{p}
$$

[1] I. Stroescu, D. B. Hume, and M. K. Oberthaler, Phys. Rev. Lett. 117, 243005 (2016).

[2] A. N. Wenz, G. Zurn, S. Murmann, I. Brouzos, T. Lompe, and S. Jochim, Science 342, 457 (2013).

[3] M. Greiner, I. Bloch, O. Mandel, T. W. Hänsch, and T. Esslinger, Appl. Phys. B 73, 769 (2001).

[4] M. A. Garcia-March, B. Juliá-Díaz, G. E. Astrakharchik, Th. Busch, J. Boronat, and A. Polls, Phys. Rev. A 88, 063604 (2013).

[5] M. A. Garcia-March and Th. Busch, Phys. Rev. A 87, 063633 (2013).

[6] H. Stoof, K. Gubbels, and D. Dickerscheid, Ultracold Quantum Fields (Springer Netherlands, Dordrecht, 2009).

[7] F. D. M. Haldane, Phys. Rev. Lett. 61, 2015 (1988).

[8] R. Islam, R. Ma, P. M. Preiss, M. Eric Tai, A. Lukin, M. Rispoli, and M. Greiner, Nature (London) 528, 77 (2015).

[9] R. Menchon-Enrich, A. Benseny, V. Ahufinger, A. D. Greentree, Th. Busch, and J. Mompart, Rep. Prog. Phys. 79, 074401 (2016).
[10] K. Bergmann, H. Theuer, and B. W. Shore, Rev. Mod. Phys. 70, 1003 (1998).

[11] K. Bergmann, N. V. Vitanov, and B. W. Shore, J. Chem. Phys. 142, 170901 (2015).

[12] N. V. Vitanov, A. A. Rangelov, B. W. Shore, and K. Bergmann, Rev. Mod. Phys. 89, 015006 (2017).

[13] K. Eckert, M. Lewenstein, R. Corbalán, G. Birkl, W. Ertmer, and J. Mompart, Phys. Rev. A 70, 023606 (2004).

[14] T. Morgan, L. J. O'Riordan, N. Crowley, B. O'Sullivan, and Th. Busch, Phys. Rev. A 88, 053618 (2013).

[15] R. Menchon-Enrich, S. McEndoo, J. Mompart, V. Ahufinger, and Th. Busch, Phys. Rev. A 89, 013626 (2014).

[16] J. Polo, A. Benseny, Th. Busch, V. Ahufinger, and J. Mompart, New J. Phys. 18, 015010 (2016).

[17] S. McEndoo, S. Croke, J. Brophy, and Th. Busch, Phys. Rev. A 81, 043640 (2010).

[18] J. Polo, J. Mompart, and V. Ahufinger, Phys. Rev. A 93, 033613 (2016). 
[19] E. M. Graefe, H. J. Korsch, and D. Witthaut, Phys. Rev. A 73, 013617 (2006).

[20] M. Rab, J. H. Cole, N. G. Parker, A. D. Greentree, L. C. L. Hollenberg, and A. M. Martin, Phys. Rev. A 77, 061602 (2008).

[21] J. L. Rubio, V. Ahufinger, Th. Busch, and J. Mompart, Phys. Rev. A 94, 053606 (2016).

[22] A. Benseny, S. Fernández-Vidal, J. Bagudà, R. Corbalán, A. Picón, L. Roso, G. Birkl, and J. Mompart, Phys. Rev. A 82, 013604 (2010).

[23] C. J. Bradly, M. Rab, A. D. Greentree, and A. M. Martin, Phys. Rev. A 85, 053609 (2012).

[24] A. Benseny, J. Gillet, and Th. Busch, Phys. Rev. A 93, 033629 (2016).

[25] M. Gajdacz, T. Opatrný, and K. K. Das, Phys. Lett. A 378, 1919 (2014).

[26] M. Gajdacz, T. Opatrný, and K. K. Das, Phys. Rev. A 83, 033623 (2011).

[27] E. Lieb and W. Liniger, Phys. Rev. 130, 1605 (1963).

[28] M. Olshanii, Phys. Rev. Lett. 81, 938 (1998).

[29] Th. Busch, B.-G. Englert, K. Rzażewski, and M. Wilkens, Found. Phys. 28, 549 (1998).

[30] K. Winkler, G. Thalhammer, F. Lang, R. Grimm, J. H. Denschlag, A. J. Daley, A. Kantian, H. P. Buchler, and P. Zoller, Nature (London) 441, 853 (2006).
[31] H. Lee, P. Kok, and J. P. Dowling, J. Mod. Opt. 49, 2325 (2002).

[32] J. Schloss, A. Benseny, J. Gillet, J. Swain, and Th. Busch, New J. Phys. 18, 035012 (2016).

[33] A. N. Boto, P. Kok, D. S. Abrams, S. L. Braunstein, C. P. Williams, and J. P. Dowling, Phys. Rev. Lett. 85, 2733 (2000).

[34] M. D. Girardeau, E. M. Wright, and J. M. Triscari, Phys. Rev. A 63, 033601 (2001).

[35] O. Zobay and B. M. Garraway, Phys. Rev. Lett. 86, 1195 (2001).

[36] T. Morgan, B. O'Sullivan, and Th. Busch, Phys. Rev. A 83, 053620 (2011).

[37] T. Schumm, S. Hofferberth, L. M. Andersson, S. Wildermuth, S. Groth, I. Bar-Joseph, J. Schmiedmayer, and P. Krüger, Nat. Phys. 1, 57 (2005).

[38] P. W. Courteille, B. Deh, J. Fortágh, A. Günther, S. Kraft, C. Marzok, S. Slama, and C. Zimmermann, J. Phys. B 39, 1055 (2006).

[39] E. Tiesinga, B. J. Verhaar, and H. T. C. Stoof, Phys. Rev. A 47, 4114 (1993).

[40] S. Inouye, M. R. Andrews, J. Stenger, H.-J. Miesner, D. M. Stamper-Kurn, and W. Ketterle, Nature (London) 392, 151 (1998).

[41] D. M. Bauer, M. Lettner, C. Vo, G. Rempe, and S. Dürr, Nature Physics 5, 339 (2009). 